\title{
BMJ Open Perceptions and attitudes of patients towards medication-related osteonecrosis of the jaw (MRONJ): a qualitative study in England
}

\author{
Andrew Sturrock, ${ }^{\odot 1}$ Philip M Preshaw, ${ }^{\circ}$ Catherine Hayes, ${ }^{3}$ Scott Wilkes ${ }^{4}$
}

To cite: Sturrock A,

Preshaw PM, Hayes C, et al. Perceptions and attitudes of patients towards medicationrelated osteonecrosis of the jaw (MRONJ): a qualitative study in England. BMJ Open 2019;9:e024376. doi:10.1136/ bmjopen-2018-024376

- Prepublication history and additional material for this paper are available online. To view these files, please visit the journal online (http://dx.doi.org/ 10.1136/bmjopen-2018-024376

Received 23 May 2018

Revised 15 December 2018

Accepted 8 January 2019

Check for updates

(C) Author(s) (or their employer(s)) 2019. Re-use permitted under CC BY-NC. No commercial re-use. See rights and permissions. Published by BMJ.

${ }^{1}$ School of Pharmacy and Pharmaceutical Sciences, University of Sunderland, Sunderland, UK

${ }^{2}$ National University Centre for Oral Health, National University of Singapore, Singapore

${ }^{3}$ Faculty of Health Sciences and Wellbeing, University of Sunderland, Sunderland, UK

${ }^{4}$ School of Medicine, University of Sunderland, Sunderland, UK

Correspondence to Andrew Sturrock; andrew.sturrock@sunderland. ac.uk

\section{ABSTRACT}

Objective To explore the impact of medication-related osteonecrosis of the jaw (MRONJ) on quality of life and to explore the attitudes and perceptions of patients towards the multidisciplinary approach to the prevention of the condition.

Design Interpretivist methodology using qualitative semistructured interviews.

Setting Primary care general medical practices and secondary care dental services in England.

Participants 23 patients; 6 with MRONJ, 13 prescribed bisphosphonates, 4 with osteoporosis not currently prescribed any medication.

Results Patients felt that MRONJ had a significant negative impact on their quality of life and had poor knowledge of the preventive strategies recommended in the literature. Patients demonstrated positive attitudes towards a multidisciplinary approach to care; however, they perceived prescribers as having the key role in articulating risk. Four salient and inter-related themes emerged from the interviews: (1) perception of knowledge, indicating limited awareness of the condition, risk factors and preventive strategies; (2) quality of life, indicating the lived experiences of patients and the physical, psychological and social impacts of MRONJ; (3) interprofessional management, indicating a perceived organisational hierarchy, professional roles and responsibilities, prioritising aspects of care, articulation of risk and communication and (4) wider context, indicating demands on National Health Service resources and barriers to dental care.

Conclusions MRONJ has a significant detrimental impact on quality of life, yet appropriate preventative education is not apparent. Effective interprofessional patient education and prevention to mitigate against the risk of developing MRONJ is required.

\section{INTRODUCTION}

Medication-related osteonecrosis of the jaw (MRONJ) is defined as exposed bone, or bone that can be probed through an intraoral or extraoral fistula, in the maxillofacial region that has persisted for more than 8 weeks in patients with a history of treatment with antiresorptive or antiangiogenic

\section{Strengths and limitations of this study}

- Although medication-related osteonecrosis of the jaw (MRONJ) is not a common finding, affected patients experience significant morbidity, and management of this condition warrants further study to improve patient care.

- There is limited research into the impact of MRONJ on patients; this is the first qualitative study that has explored the perceptions and consequent attitudes of patients and the resultant impact of the condition on quality of life.

- A qualitative method yielded rich data through indepth semistructured interviews with three groups of patients (patients with a diagnosis of MRONJ, patients prescribed bisphosphonates and patients with a diagnosis of osteoporosis who are not currently prescribed medication). Constant comparison with concurrent data collection and analysis allowed further exploration and refining of emergent themes.

- The study was based around an a priori assumption of limited knowledge among patients in relation to MRONJ; patients were provided a patient information leaflet in advance, therefore exposing participants to the concepts before the interview.

drugs, and where there has been no history of radiation therapy to the jaw or no obvious metastatic disease to the jaws. ${ }^{1}$ The risks for MRONJ are hypothesised to be related to the unique nature of the blood supply, and the anatomical structure and function of the jaw bones. $^{2-6}$

A number of drugs that are indicated for use in osteoporosis, Paget's disease or the treatment of cancer have been associated with MRONJ. These include both oral and intravenous bisphosphonates such as alendronic acid or zoledronate, receptor activator of nuclear factor kappa-beta ligand inhibitors such as denosumab, and antiangiogenic drugs such as bevacizumab, sunitinib and aflibercept. ${ }^{7}$ In practice, the most commonly prescribed 
agents are oral bisphosphonates for the management of osteoporosis.

The major risk factor for the development of MRONJ is a dental extraction in a patient exposed to implicated medicines $^{8}$; however duration of therapy with antiresorptive drugs exceeding 4 years and concomitant administration of corticosteroids are also associated with an increased risk. ${ }^{1}$ Exact incidence and prevalence rates of MRONJ are unclear, with varying reports in the literature. $^{4-6}$ The estimated incidence of MRONJ in patients with cancer treated with antiresorptive or antiangiogenic drugs is $1 \%$ and in patients with osteoporosis treated with antiresorptive drugs is $0.01 \%-0.1 \% .^{7}$

A systematic review of the diagnosis and management of osteonecrosis of the jaw identified the elimination or stabilisation of oral disease before initiating antiresorptive agents as a preventative strategy for MRONJ. ${ }^{9}$ Several prospective studies have identified that dental screening and preventive strategies reduce the risk of osteonecrosis of the jaw. ${ }^{111}$ Before commencement of drugs associated with MRONJ, or as soon as possible thereafter, patients should be supported in becoming as dentally healthy as possible. This aim is to prioritise care that will reduce mucosal trauma and/or act prophylactically to help avoid subsequent dental extractions or conditions, which may further predispose the patient to surgical or dental procedures that further impact on the osseous structures of the jaw. ${ }^{7}$

Clinical guidelines published by the Scottish Dental Clinical Effectiveness Programme recommend that patients with high-risk oncology should undergo a thorough dental assessment, with necessary dental treatment prior to the initiation of drug therapy. ${ }^{7}$ Guidance for prescribers and pharmacists also recommends that patients (and/or their carers) are advised that there is a risk of MRONJ, but should ensure that they understand that the risk is small. Patients should be advised to make an appointment with their dentist to ensure they are dentally fit and inform their dentist that they will be taking the prescribed medication. ${ }^{7}$

A multidisciplinary approach to the prevention of MRONJ is recommended in the literature, incorporating both patient and health professional education on the risk of the development of MRONJ, appropriate preventive measures and oral health instruction. ${ }^{72-16}$ Our recent qualitative study of general medical practitioners (GMPs) and pharmacists in North East England found that both professional groups had limited knowledge and awareness of MRONJ and due to the complex medical histories of patients, practitioners often overlooked the advice related to the risk and prevention of MRONJ. ${ }^{17}$

The aim of this study was to explore the impact of osteonecrosis of the jaw on patients, and to explore the attitudes and perceptions of these patients towards the multidisciplinary approach to the prevention of MRONJ. While several drugs are thought to contribute to the aetiology of MRONJ, this study focused specifically on the association between bisphosphonates and osteonecrosis of the jaw and the multidisciplinary approach to the prevention of this rare, yet serious, adverse effect.

\section{Aims}

1. To explore the perceived impact of MRONJ on patients with a diagnosis of the condition.

2. To explore the attitudes and perceptions of patients towards the roles of the pharmacist, GMP and dentist in the prevention of MRONJ.

3. To explore the barriers and enablers to optimise risk prevention of MRONJ.

\section{METHODS \\ Design}

A grounded theory approach was used throughout this research. ${ }^{18}$ Constant comparison was utilised as a means of enriching the data through iterative data collection and analysis; the emergence of themes during the process provided the opportunity for further exploration during subsequent data collection. ${ }^{19}$

An initial topic guide (online supplementary document 1) was developed by the principal investigator based on the published literature and the findings of our previous qualitative study. ${ }^{17}$ The topic guide was reviewed and refined by the multidisciplinary research team and served as a benchmark for semistructured one-to-one interviews carried out at the participant's home, general medical practice or dental clinic. The interviews were audio-recorded and transcribed verbatim to aid qualitative analysis.

\section{Participants}

Participants were recruited with the assistance of three National Institute for Health Research Clinical Research Networks (NIHR CRNs); North East and North Cumbria, Yorkshire and Humber, and North Thames. Three distinct groups were recruited to the study: (1) patients prescribed bisphosphonates, (2) patients with a diagnosis of osteoporosis not currently undergoing drug treatment and (3) patients with a diagnosis of MRONJ. An invitation letter (online supplementary documents 2-4) and participant information sheet (online supplementary documents 5-7) were posted to patients in groups 1 and 2 by their GMP and a convenience sample of participants who responded to the invitation was implemented. Participants were assigned a participant number to ensure anonymity.

Patients in group 3 (diagnosis of MRONJ) were recruited through the Oral and Dental Specialty Group of the NIHR CRNs; two secondary care dental hospitals recruited participants by posting invitation letters and participant information sheets to eligible patients.

\section{Analysis}

Constant comparison allowed for enrichment of data and for new concepts to be explored through subsequent interviews; Ritchie and Spencer's framework analysis $(2002)^{20}$ allowed salient themes to be identified from the data. Framework analysis involved a five-stage process: familiarisation with the data; development of a thematic 
Table 1 Participant characteristics

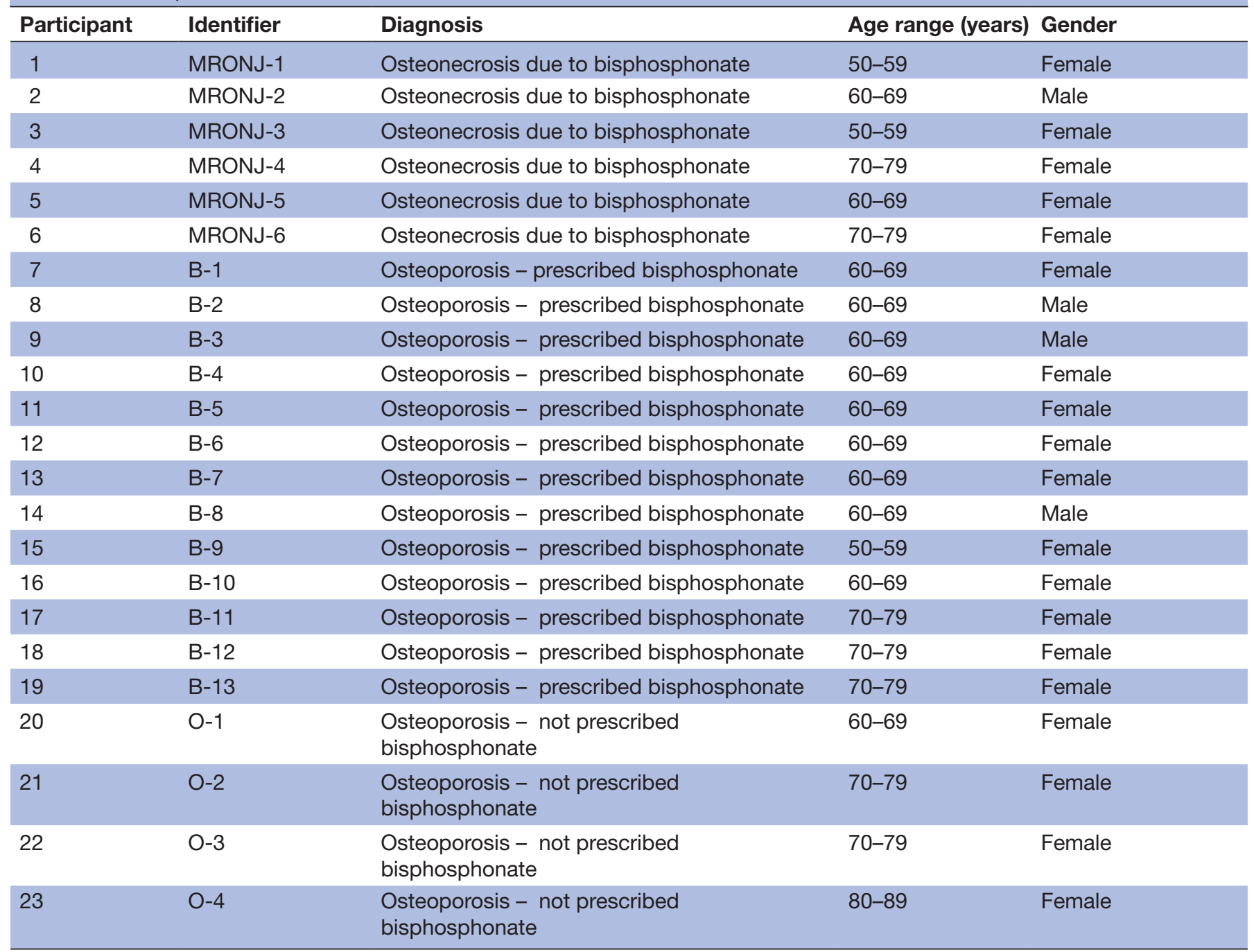

Four salient inter-related themes emerged from the data: (1) perceptions of knowledge; (2) quality of life; (3) interprofessional management and (4) wider context.

MRONJ, medication-related osteonecrosis of the jaw.

framework; indexing data; charting of the data and mapping of the data. Themes were reviewed until definitive concepts could be produced from the data.

\section{Patient involvement}

The principal investigator met with a patient representative from the University of Sunderland Patient, Carer and Public Involvement Group to discuss the design and ethical implications of the study. This included the co-constructed design of the patient information sheet, ensuring informed consent and finally information regarding the opportunity to access further advice or support following their participation in the study.

\section{RESULTS}

In all, 23 patients were recruited to this study (table 1). In-depth semistructured interviews were carried out between May 2017 and March 2018 until no new themes emerged and current ones were exhausted. Interviews took place in patient's homes, at their general medical practice or at their secondary care dental clinic; 1 hour was designated for each interview.

\section{Perceptions of knowledge}

The concept of MRONJ was introduced in the participant information sheet and opened up for further discussion during the interview; participants without a diagnosis of MRONJ had minimal awareness of the associated risk.

They didn't explain about- anything about any side-effects or anything about trouble with your teeth. (B-6)

Those patients with a diagnosis of MRONJ were aware of the condition and how this was related to their prescribed medication. All patients with MRONJ stated that they were unaware of this risk prior to commencing treatment with the bisphosphonate. 
I was given no information about that...Doctors don't tell you about the side-effects of drugs. (MRONJ-6)

Most patients reported that information relating to the risk and preventive strategies for MRONJ complications had not been discussed with the prescriber or pharmacist on initiation. Where patients had awareness of these issues, the information was typically gained from the patient information leaflet supplied with their medication.

Well, I usually read the little leaflet for any, you know, side-effects that they might have. (B-8)

It was clear from the discussions that the patients prescribed a bisphosphonate were uncertain about required duration of therapy; many patients had been prescribed the drugs for a number of years but were unclear on whether therapy should be continued indefinitely or for a set period of time.

I reckon I've been taking it more than five years now. And it should- I've got a feeling it should've been reviewed after five years. (B-8)

Patients felt that although the internet can provide access to information, due to age, many people in this patient group have limited knowledge of, or access to, web-based information.

You know, it's only since the internet that people able to look up on the actual - I mean, I- I'm not - I do use the internet, but not often or very well- I'm not on it every day cos I don't have it where I live. (MRONJ-6)

\section{Quality of life}

Most of the patients interviewed had a complex medical history. The age of participants and the presence of comorbidities meant that osteoporosis was typically one of a number of ongoing medical conditions for which they were undergoing treatment; as a result, most patients were prescribed a number of medications.

At one time, when I first came to hospital, I was on twenty- about twenty tablets a day, you know, which is too much. (B-2)

Participants with a diagnosis of MRONJ highlighted the impact that the condition has had on their quality of life. Participants described experiencing a significant amount of pain with the condition, requiring the frequent use of analgesic medication.

The big problem is all my lips are tender. When I touch them, it - it's just as though - I've never been hit in face, but- but I can imagine somebody hitting you in the face. I can imagine it feeling like that. And - and the tenderness, it never goes. It's always there. I touch it and I feel as though I don't want to touch it. (MRONJ-2)
Participants identified challenges in relation to eating and drinking, and the associated social anxiety of eating awkwardly in public.

Psychological and mental, yeah. If you're going out to a restaurant, then you have to be very careful. You don't want people to see that you are eating awkwardly. (MRONJ-5)

The psychological implications of a diagnosis of MRONJ were highlighted by participants; these were seen to take less of a priority for healthcare professionals but have a significant impact on the quality of patients' lives.

This is difficult, but mentally, it gives you some kind of anxiety because you- you- you know your bone is there - a little piece of bone on your left-hand side is there. (MRONJ 5)

All participants with a diagnosis of MRONJ were required to attend secondary care dental hospitals, where their condition was managed and regularly reviewed. In some cases, patients had to travel a considerable distance for treatment and were required to attend frequent appointments in secondary care.

I mean, I go every month at the moment, it's quite an- a big impact, I guess, in terms of appointments. Well, they- they have a look, see if it's got any worse, and then record it. They often have to send me for more x-rays. (MRONJ-2)

Participants with a diagnosis of MRONJ expressed concerns regarding the potential complications of the disease, the need for antibiotic treatment and for surgical intervention.

I have had to have lots of antibiotics, it seems to keep getting infected. Hopefully they will keep working, but one time, they had to give me some extra strong antibiotics because the normal antibiotics didn't work. (MRONJ-2)

You think, maybe perhaps in the future, you need to have an operation. It's a big operation. (MRONJ-5).

\section{Interprofessional management}

It became clear from the interviews that participants perceived there to be a clear organisational hierarchy in terms of the management of their condition. Participants felt that it was the responsibility of the prescribing clinician to provide information relating to the adverse effects of medication.

I think - and you needed that information, I think it should be the doctors telling you when- when he prescribes it, to say to - as a precaution, you should go to your dentist. (O-3)

Most participants placed trust in the professionals managing their care and perceived that prescribers would have already utilised professional judgement in relation to the possible risks and benefits of medication. 
I'm sure the doctor will use his own discretion, you know. That it is safe and appropriate. (B-2)

However, many participants identified that the risks and benefits of medication are not well articulated to patients, making it difficult to make informed decisions around their care.

I think they should be able to provide the risks and the benefit and discuss with the patient what's probably be-best with them. I don't think this is done very well. (MRONJ-6)

Due to the complexity of patient's medical profiles and the associated polypharmacy, it was identified that information is typically prioritised and that healthcare professionals only have limited time to provide information.

They haven't got the time to go through everything with you. [chuckles] I think they have to pick out the key things. (B-1)

Participants perceived pharmacists to have an important role in the reinforcement of advice given by prescribers and were receptive to receiving information from pharmacists relating to the administration and potential adverse effects of medication.

Quite often, you know, you talk to your GP and you go away and you just forget- you forget something that they've said. So, having it reinforced a couple of times I think's a good idea. (B-8)

Pharmacists were seen as having specialised knowledge in relation to the adverse effects of medicines; a number of patients had experienced a formal medication review by their pharmacist and appreciated the opportunity to discuss their medication and adverse effects.

I feel as though the pharmacist that I go to, I could ask her anything and she would tell us. I have had a review with her, she's very, very helpful and knowledgeable about medication. (B-5)

Participants reported that dental practitioners routinely ask about changes to prescribed medicines during check-up and treatment appointments. Some participants identified that their dentist specifically asks about their prescribed bisphosphonate, but the interest in these drugs had not been explained to the participants.

You've got to fill a- a form in every time with your medicines on. And funnily enough, alendronic acid is the one that I often forget and miss off. And they have asked us 'are you still taking that?' (B-1)

Participants discussed the need for good communication between the professional groups to support the prevention of MRONJ. Participants were all happy for information to be shared between the professions and expected information regarding their treatment to be communicated effectively.
If the doctor has recommended me to go, I would think there should be at least some liaison with the dentist and the doctors and that was on your medical records to say you're getting that check done. (O-3)

Participants also described the importance of taking responsibility for their own actions. If provided with information or management advice, they perceived they ought to have ensured that this was acted on. Participants did stress that in order to take personal responsibility, they needed to be appropriately informed by the healthcare professional(s).

It's your own responsibility. If you've been told about something properly, you know, it's then your responsibility too. You've got to look after yourself, you know. (B-2)

\section{Wider context}

Participants identified that there is an increasing demand on National Health Service (NHS) resources and perceived that all healthcare professionals have a heavy workload. As such, they felt that the implementation of preventive strategies could potentially place more demands on staff time and the already limited appointment schedules.

Doctors are so over-stretched and - and - you only have a short time for the appointment to get the information. Sometimes you still wait forever to even get an appointment. (MRONJ-6)

Although most of the participants had a history of regular dental appointments, there was a strong feeling that many patients have a general reluctance to seek dental advice. Potential barriers such as a phobia of dental treatment, a perceived lack of awareness of oral health and the financial implications of dental treatment were all identified by participants.

Terrified. Uh-huh. Always have been. (B-5)

You have to pay for the examination and then obviously, depending on the amount of work that you need, that can be quite expensive. And not everybody has that money. (B-6)

\section{DISCUSSION}

MRONJ is a serious condition that requires complex management, and current literature is indicative of the importance of preventive care interventions, due to the subsequent associated morbidity and challenges in treating osteonecrosis of the jaw. ${ }^{1}$ In this study, it was apparent that MRONJ has a significant effect on the quality of life experienced by patients who were interviewed. Previously, a study of 34 patients with MRONJ utilising the Oral Health Impact Profile questionnaire-14 found that the condition significantly affects the quality of life. ${ }^{21}$ While this provides tangible metrics regarding the significance of the condition of patients, it provides 
no specific information on what this means to people in their daily lives. The qualitative insight generated by our study has provided the first documented experiences of this particular patient group, highlighting the significant issues they face and the ongoing physical, psychological and social distress they associate with the condition.

As MRONJ can, in many cases, be prevented with appropriate oral health education and preventive care, the importance of such measures should be stressed to all healthcare professionals managing this particular patient group. It also leads us to consider how other allied health professionals may also incorporate the importance of this into their practice with patients and their carers and families. Masson ${ }^{22}$ et alidentified that only $11.8 \%$ of GMPs and $9.7 \%$ of pharmacists advised patients to inform their dentist they were using a bisphosphonate. ${ }^{22}$ Our previous qualitative study of GMPs and pharmacists in England also identified limited knowledge among these professional groups in relation to the risk and prevention of MRONJ. ${ }^{17}$

Patients from all three groups were generally unaware of the risks and preventive strategies, and the patients with MRONJ reported limited knowledge prior to diagnosis. A quantitative study $(\mathrm{n}=55)$ found that the majority of patients acquired knowledge about the drug they were prescribed from patient information leaflets $(62 \%)$, with few patients $(13 \%)$ receiving this information from their GMP. When asked to identify side effects of bisphosphonate therapy, only $32 \%$ of patients receiving IV, and $17 \%$ patients receiving oral, bisphosphonates were aware of the risk of developing osteonecrosis of the jaw. ${ }^{23}$ When patients in our study possessed some knowledge, this typically came from the information leaflet supplied with medication or from the internet.

Although published clinical guidelines recommend that patients should be referred for dental assessment and treatment prior to initiation of bisphosphonate therapy, ${ }^{7}$ it is apparent this is not happening in practice. A lack of knowledge in relation to the risk and appropriate preventative strategies by prescribers is potentially exposing patients to a condition with significant quality of life implications and represents a key medication safety issue. An awareness of MRONJ among prescribers is a key to ensuring that an appropriate risk assessment can be made relative to the prescribing of implicated medicines and the need for the effective education of patients on preventative strategies.

Patients prescribed bisphosphonates were confused about the intended duration of treatment with the drug; some patients were aware that the medication would only be prescribed for a set duration of time, whereas for others, this medication had already been prescribed for many years without any evident review.

Participants described a perceived organisational hierarchy in relation to the management of their health; they expected prescribers to use professional judgement on the suitability of the medication for them and to provide information related to the adverse effects of medications.
Many of the participants interviewed have complex medication histories, live with comorbid conditions and as a consequence are simultaneously prescribed multiple medications. Participants therefore described the need for prescribers to prioritise information related to their clinical management and in-patient education in relation to their polypharmacy.

Participants perceived that the pharmacist has an important role in reinforcing advice and were positive in their regard of the pharmacist's role in providing information on medications and conducting medication reviews. Participants reported that their general dental practitioners were active in recording medication details and were also receptive to information being shared between medical and dental services. Key barriers in relation to the multidisciplinary prevention of MRONJ, such as heavy demands on NHS resources, attitudes towards oral health, a reluctance to attend dental appointments and the financial issues associated with dental care, were all identified by participants.

This study has explored the attitudes and perception of patients prescribed bisphosphonates, focusing on those with a diagnosis of osteoporosis. The literature is clear that the incidence of osteonecrosis is greater in patients prescribed intravenous bisphosphonates for the treatment of cancer; further work exploring the management of this patient group and any variation in the attitudes towards risk and ongoing management would substantially add to this body of literature.

Patients have already demonstrated positive intentions to change oral health behaviours following pharmacy-based oral health interventions ${ }^{24}$; further work to explore the role of the pharmacist in the interprofessional prevention of MRONJ should be considered. Patients in our study described the benefit of formal medication reviews with their pharmacist and a willingness to engage with pharmacy services to receive information related to the adverse effects of medication. Both the Medication Use Review (MUR) and New Medicine Services (NMS) are advanced services within the NHS Community Pharmacy Contractual Framework in England. An MUR is a structured, adherence-centred polypharmacy review of patients prescribed multiple medicines and the NMS service provides support for patients with long-term conditions that have been newly prescribed a medicine. ${ }^{25} 26$ However, the MUR and NMS service specifications do not currently include bisphosphonates; the inclusion of this group of drugs could provide an opportunity for reinforcement of preventative advice during the initiation stages of treatment with bisphosphonates.

The perspective of dental practitioners on how the multidisciplinary team can collaborate to improve patient care would be important to consider before implementing any preventative strategies. A recent publication in British Dental Journal emphasised the potential benefits of interprofessional working between pharmacy and dental professionals ${ }^{27}$; further work to develop such services could be of particular benefit to this patient group. 


\section{CONCLUSION}

MRONJ has a significant detrimental impact on patient quality of life, with significant physical, psychological and social implications. However, patients demonstrated limited knowledge of these risks and of the preventive strategies recommended for their avoidance, in the literature.

Patients perceive prescribers to be responsible for educating them on the risks associated with medications. The formal role of the pharmacist, however, can provide a significant opportunity to reinforce information and provide advice to patients regarding both newly prescribed medications and the evaluation of their other pre-existing pharmacological regimes, via formal medication reviews.

Increased focus on preventative dental care with the education of other healthcare professionals and patients on the importance of oral health and preventative strategies could potentially improve patient safety and prophylactically reduce the risk of the development of MRONJ in practice.

Acknowledgements We thank the participants who generously gave their time, the support staff from the NIHR CRNs and the clinicians and practice staff from the general medical practices and secondary care dental services.

Contributors AS, SW, CH and PMP designed the study. AS recruited the participants and carried out the study. AS identified the thematic framework and interpreted the data. AS, SW, PMP and CH designed the qualitative research methodology and reviewed and refined the data. AS wrote the paper and all authors revised it. AS received training in qualitative research skills by the research team and through attendance at a Qualitative Research Methods in Health Course at University College London.

Funding This work was supported by the 2016 Pharmacy Research UK and the United Kingdom Clinical Pharmacy Association Research Award.

Competing interests None declared.

Patient consent for publication Not required.

Ethics approval Ethical approval was obtained from the NHS North East - York Research Ethics Committee (REF: 17/NE/0033).

Provenance and peer review Not commissioned; externally peer reviewed.

Data sharing statement Participant information sheets and invitation letters are included (Supplementary Files 2-7); no further data shared.

Open access This is an open access article distributed in accordance with the Creative Commons Attribution Non Commercial (CC BY-NC 4.0) license, which permits others to distribute, remix, adapt, build upon this work non-commercially, and license their derivative works on different terms, provided the original work is properly cited, appropriate credit is given, any changes made indicated, and the use is non-commercial. See: http://creativecommons.org/licenses/by-nc/4.0/.

\section{REFERENCES}

1. Ruggiero SL, Dodson TB, Fantasia J, et al. American Association of Oral and Maxillofacial Surgeons position paper on medicationrelated osteonecrosis of the jaw-2014 update. J Oral Maxillofac Surg 2014;72:1938-56.

2. Medicines Healthcare Regulatory Agency. Drug Safety Update: Bisphosphonates: osteonecrosis of the jaw. 2009 https://www.gov. uk/drug-safety-update/bisphosphonates-osteonecrosis-of-the-jaw (Accessed 2 Apr 2018).

3. Nakajima K, Tagaya A, Otonari-Yamamoto M, et al. Composition of the blood supply in the sublingual and submandibular spaces and its relationship to the lateral lingual foramen of the mandible. Oral Surg Oral Med Oral Pathol Oral Radiol 2014;117:e32-8.

4. Bernardi S, Mummolo S, Ciavarelli LM, et al. Cone beam computed tomography investigation of the antral artery anastomosis in a population of Central Italy. Folia Morphol 2016;75:149-53.

5. Bernardi S, Bianchi S, Continenza MA, et al. Frequency and anatomical features of the mandibular lingual foramina: systematic review and meta-analysis. Surg Radiol Anat 2017;39:1349-57.

6. Gray H. Gray's anatomy: the anatomical basis of medicine and surgery. 39th edn. London: Churchill Livingston Elsevier, 2004.

7. Scottish Dental Clinical Effectiveness Programme. Oral Health management of patients at risk of medication-related osteonecrosis of the jaw. Dental clinical guidance. Scottish Dental Clinical Effectiveness Programme: Dundee, 2017.

8. Bernardi S, Di Girolamo M, Necozione S, et al. Antiresorptive drugrelated osteonecrosis of the jaws literature review and 5 years of experience Musculoskelet Surg. 2018 https://doi.org/

9. Khan AA, Morrison A, Hanley DA, et al. Diagnosis and management of osteonecrosis of the jaw: a systematic review and international consensus. J Bone Miner Res 2015;30:3-23.

10. Dimopoulos MA, Kastritis E, Bamia C, et al. Reduction of osteonecrosis of the jaw (ONJ) after implementation of preventive measures in patients with multiple myeloma treated with zoledronic acid. Ann Oncol 2009;20:117-20.

11. Vandone AM, Donadio M, Mozzati M, et al. Impact of dental care in the prevention of bisphosphonate-associated osteonecrosis of the jaw: a single-center clinical experience. Ann Oncol 2012;23:193-200.

12. Shannon J, Shannon J, Modelevsky S, et al. Bisphosphonates and osteonecrosis of the jaw. J Am Geriatr Soc 2011;59:2350-5.

13. Patel V, McLeod NM, Rogers SN, et al. Bisphosphonate osteonecrosis of the jaw-a literature review of UK policies versus international policies on bisphosphonates, risk factors and prevention. Br J Oral Maxillofac Surg 2011;49:251-7.

14. European Medicines Agency. CHMP Assessment report on bisphosphonates and osteonecrosis of the jaw, london: european medicines agency]. 2009. http://www.ema.europa.eu/docs/en_GB/ document_library/Report/2010/01/WC500051428.pdf (Accessed 2 Apr 18).

15. Rayman S, Almas K, Dincer E. Bisphosphonate-related jaw necrosis: a team approach management and prevention. Int J Dent Hyg 2009;7:90-5.

16. Gaudin E, Seidel L, Bacevic M, et al. Occurrence and risk indicators of medication-related osteonecrosis of the jaw after dental extraction: a systematic review and meta-analysis. J Clin Periodontol 2015;42:922-32.

17. Sturrock A, Preshaw PM, Hayes C, et al. Attitudes and perceptions of GPs and community pharmacists towards their role in the prevention of bisphosphonate-related osteonecrosis of the jaw: a qualitative study in the North East of England. BMJ Open 2017;7:e016047.

18. Glaser BG, Strauss AL. The discovery of grounded theory: strategies for qualitative research. Chicago: Aldine, 1967.

19. Boeije H. A purposeful approach to the constant comparative method in the analysis of qualitative interviews. Quality and Quantity 2002;36:391-409.

20. Ritchie J, Spencer L. Qualitative data analysis for applied policy research. In: Humberman M, Miles M, eds. The qualitative researcher's companion. Thousand Oaks: Sage, 2002:305-29.

21. Miksad RA, Lai KC, Dodson TB, et al. Quality of life implications of bisphosphonate-associated osteonecrosis of the jaw. Oncologist 2011;16:121-32.

22. Masson D, O'Callaghan E, Seager M. The knowledge and attitudes of North Wales healthcare professionals to bisphosphonate associated osteochemonecrosis of the jaws. J Disab Oral Health 2009;10:175-83.

23. Bauer JS, Beck N, Kiefer J, et al. Awareness and education of patients receiving bisphosphonates. J Craniomaxillofac Surg 2012;40:277-82.

24. Sturrock $A$, Cussons $H$, Jones $C$, et al. Oral health promotion in the community pharmacy: an evaluation of a pilot oral health promotion intervention. Br Dent J 2017;223:521-5.

25. Pharmaceutical Services Negotiating Committee. Medicines use review. http://psnc.org.uk/services-commissioning/advancedservices/murs/ (Accessed 2 Apr 2018).

26. Pharmaceutical Services Negotiating Committee. New medicines service. http://psnc.org.uk/services-commissioning/advancedservices/nms/ (Accessed 2 Apr 2018).

27. Wilson N, Soni A. Interprofessional working: a spearhead opportunity for dentistry and pharmacy. Br Dent J 2016;221:607-8. 